\title{
Hybrid Waves Guided by Ultrathin Films
}

\author{
Lluís Torner, Member, IEEE, Juan P. Torres, Concepción Ojeda, and Dumitru Mihalache
}

\begin{abstract}
The existence conditions and waveguiding properties of new hybrid, TE-dominant waveguide modes of properly tailored waveguides consisting on ultrathin films cladded by a positive birefringent crystal are analyzed. The results show that the existence conditions of such waves strongly depend on the waveguide parameters. Accordingly, the related guided-toradiated wave transition holds promise for switching applications requiring a strong sensitive dependence on the material refractive index values.
\end{abstract}

\section{INTRODUCTION}

W AVES guided by planar structures containing low-loss dielectric birefringent media have been investigated since the early days of Integrated Optics, due to the fact that the involved technology usually employs uniaxial crystalline materials, such as lithium niobate and lithium tantalate: Crystal optical anisotropy plays a decisive role in many integrated optical devices, for instance to obtain TE-TM mode conversion or to achieve phase-matching condition for second-harmonic generation and other nonlinear parametric interactions, and gives rise to the existence of hybrid guided waves. Totally guided, as well as leaky waves guided by such structures have been extensively analyzed, hence now dispersion properties of known waveguide modes are well established (for a review see [1], [2], and references therein). Nevertheless, properly tailored slab waveguides with positive birefringent cladding media allow existence of new totally guided waves for waveguide parameters below usual cutoff [3], [4], which, to our knowledge, have not been noticed early. Our aim here addresses these new waveguide modes.

We pay attention to a thin film of thickness $D$ of an isotropic material with refractive index $n_{f}$, cladded by an isotropic medium with refractive index $n_{c}$ and a positive birefringent crystal, whose ordinary and extraordinary refractive indices are $n_{o s}$ and $n_{e s}$. Integrated optical technology typically employs dielectric materials for which $n_{e s}>n_{o s}>n_{c}$ (e.g., glasscladded $\mathrm{LiTaO}_{3}$-based waveguides). Such structures allow existence of both TE-TM and hybrid waveguide modes but, as it is well-known, there is a cutoff waveguide thickness for the guided waves to exist. On the contrary, properly tailored waveguides having $n_{e s}>n_{c}>n_{o s}$, exhibit no cutoff for a totally guided wave to exist [3]. Above usual cutoff these waves become the well-known hybrid guided modes,

Manuscript received March 23, 1993. This work was supported by the Comisión Interministerial de Ciencia y Tecnología of the Spanish Government under grant TIC92-0094-C02-02.

L. Torner, J. P. Torres, and C. Ojeda are with the Department of Signal Theory and Communications, Polytechnic University of Catalonia, 08080 Barcelona, Spain.

D. Mihalache is with the Department of Theoretical Physics, Institute of Atomic Physics, Bucharest, Romania.

IEEE Log Number 9414403. and for $D / \lambda \rightarrow 0$, with $\lambda$ being the wavelength of the used radiation, they become the surface waves guided by the interface between the dielectric materials forming the substrate and the cover, which have been pointed out by D'yakonov [4].

Our goal in this paper is to investigate the existence conditions and waveguiding properties of the above-mentioned hybrid waves guided by ultrathin films. Here we will deal with a very simple structure, which will enable us to clearly point out the nature and properties of the allowed guided waves. More complex configurations exhibiting analogous phenomena will be treated elsewhere. Organic materials, with engineered optical properties, hold promise for applications in integrated optical, both passive and nonlinear, second and third-order, devices [5], [6]. Thus, when going to the results, we will take the typical parameters of a glass-cladded organic film $\left(n_{c} \simeq\right.$ $\left.1.55, n_{f} \simeq 1.57\right)$, with a crystal quartz substrate $\left(n_{o} \simeq 1.547\right.$, $n_{e} \simeq 1.556$ ), operating at $\lambda=0.532 \mu \mathrm{m}$. We will focus our attention to film thicknesses ranging from $50-150 \mathrm{~nm}$, which correspond to the typical values of Langmuir-Blodgett ultrathin films.

This paper is organized as follows. Waveguide configuration and general field and modal properties are outlined in Section II. In Section III the eigenvalue equation of the allowed hybrid guided waves is obtained, and the critical conditions defining the cutoff optical axis orientation for the guided waves to exist are established. The waveguiding properties of the allowed guided waves are analyzed in Section IV. Attention is focused on the possibility of tuning the value of the cutoff optical axis orientation through the waveguide parameters, and on the nature of the allowed hybrid guided waves in terms of transversal and longitudinal field components. Finally, in Section V we summarize our main conclusions.

\section{WAVEGUIDE CONFIGURATION}

The optical waveguide we will consider here is shown in Fig. 1. The optical axis of the birefringent crystal forming the substrate is assumed to lie on the guide plane, forming an angle $\theta$ with the waveguide axis, which corresponds to off-axis propagation in an $X$ or $Y$-cut sample. The wave propagation direction is taken along the $x$-axis and a timeharmonic dependence has been assumed, so that the fields at any point have the form $\exp [j(\beta x-\omega t)]$, with $\beta$ being the propagation constant. For a guided wave $\mathcal{E}_{z}, \mathcal{H}_{x}$, and $\mathcal{H}_{y}$ are $\pi / 2$ out of phase with each other field component, hence to avoid unnecessary complex quantities we define $\overrightarrow{\mathcal{E}}=\left(E_{x}, E_{y}, j E_{z}\right)$, and $\overrightarrow{\mathcal{H}}=\left(j H_{x}, j H_{y}, H_{z}\right) / \eta$, with $\eta$ being the characteristic impedance of free-space.

In the coordinate system $(X, Y, Z)$ that coincides with the principal axis of the uniaxial crystal forming the substrate, the 


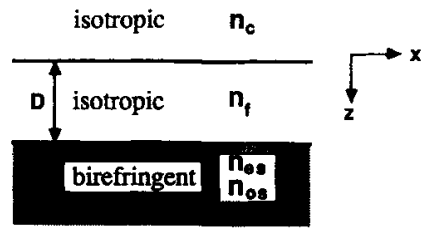

Fig. 1. Schematic waveguide structure.

relative dielectric tensor is diagonal and takes the form

$$
[\epsilon]_{X Y Z}=\left(\begin{array}{ccc}
n_{o s}^{2} & & \\
& n_{o s}^{2} & \\
& & n_{e s}^{2}
\end{array}\right) .
$$

For an arbitrary orientation of the optical axis, the dielectric tensor is obtained by application of the appropriate rotation operator to (1). For the orientation considered here one gets a symmetric tensor whose nonvanishing elements write

$$
\begin{aligned}
\epsilon_{x x} & =n_{o s}^{2} \sin ^{2} \theta+n_{e s}^{2} \cos ^{2} \theta \\
\epsilon_{y y} & =n_{o s}^{2} \cos ^{2} \theta+n_{e s}^{2} \sin ^{2} \theta \\
\epsilon_{z z} & =n_{o s}^{2} \\
\epsilon_{x y} & =\left(n_{e s}^{2}-n_{o s}^{2}\right) \sin \theta \cos \theta .
\end{aligned}
$$

In general, a waveguide such as the former one can only support hybrid modes, with the six field components. In the limiting cases $\theta=0^{\circ}$ ( $X, Y$-cut; $Z$-propagating) and $\theta=$ $90^{\circ}$ ( $X, Y$-cut; $Y, X$-propagating), the allowed guided modes break down in the decoupled TE and TM sets.

\section{HYBRID GUIDED MODES}

\section{A. Eigenvalue Equation}

We are looking for totally guided waves, therefore the fields in the cover and in the substrate must be evanescent. Since the substrate consists of a birefringent crystal, the field in this region will be a weighted superposition of ordinary and extraordinary waves, hence

$$
\begin{aligned}
\vec{E}_{s}(z) & =\vec{E}_{o s} \exp \left[k_{0} \gamma_{o s}(D-z)\right] \\
& +\vec{E}_{e s} \exp \left[k_{0} \gamma_{e s}(D-z)\right]
\end{aligned}
$$

with $k_{0}$ being the free-space wave number. The transversal decay constants $\gamma_{o s}$ and $\gamma_{e s}$ come from the wave equation

$$
\nabla^{2} \vec{E}+k_{0}^{2}[\epsilon] \vec{E}=\nabla(\nabla \cdot \vec{E}) .
$$

One gets

$$
\begin{aligned}
\gamma_{o s} & =\left[N^{2}-n_{o s}^{2}\right]^{1 / 2} \\
\gamma_{e s} & =\frac{n_{e s}}{n_{e s}(\theta)}\left[N^{2}-n_{e s}^{2}(\theta)\right]^{1 / 2}
\end{aligned}
$$

where $N \equiv \beta / k_{0}$ is the effective index of guided modes and

$$
\frac{1}{n_{e s}^{2}(\theta)}=\frac{\sin ^{2} \theta}{n_{e s}^{2}}+\frac{\cos ^{2} \theta}{n_{o s}^{2}}
$$

with $n_{e s}(\theta)$ being the refractive index of the extraordinary wave propagating in an unbounded uniaxial crystal. In the isotropic cover the ordinary and the extraordinary solutions degenerate, thus $\vec{E}_{c}(z)=\vec{E}_{c} \exp \left[k_{0} \gamma_{c} z\right]$, with

$$
\gamma_{c}=\left[N^{2}-n_{c}^{2}\right]^{1 / 2} \text {. }
$$

Existence of (5) and (6) indicates that all the field components in (3) can be expressed in terms of two arbitrary constants. Accordingly, using a matrix notation to render the expressions in a compact form, the electric field in the substrate can be written as

$$
\begin{aligned}
\vec{E}_{\boldsymbol{s}}(z)= & {\left[\begin{array}{cc}
-\tan \theta & 1 \\
1 & \frac{-n_{o s}^{2} \tan \theta}{\gamma_{o s}^{2}} \\
\frac{-N \tan \theta}{\gamma_{o s}} & \frac{N \gamma_{e s}}{\gamma_{o s}^{2}}
\end{array}\right] } \\
& \cdot\left[\begin{array}{l}
E_{o s} \exp \left[k_{0} \gamma_{o s}(D-z)\right] \\
E_{e s} \exp \left[k_{0} \gamma_{e s}(D-z)\right]
\end{array}\right] .
\end{aligned}
$$

In the cover, the double solution (8) yields an expression analogous to (9), but for the TE and the TM field components. One arrives at

$$
\vec{E}_{c}(z)=\left[\begin{array}{cc}
0 & 1 \\
1 & 0 \\
0 & \frac{-N}{\gamma_{c}}
\end{array}\right]\left[\begin{array}{l}
E_{o c} \\
E_{e c}
\end{array}\right] \exp \left[k_{0} \gamma_{c} z\right]
$$

The eigenvalue equation for guided waves is obtained by jointing (9) and (10) with the fields in the film region. This can be easily done by means of the characteristic-matrix formalism. Following Berreman approach [7], the characteristic matrix, $T$, of a dielectric film of thickness $D$ is defined as

$$
\Psi(z=D)=T \Psi(z=0)
$$

with $\Psi(z)$ being an ordered four-dimensional array containing the tangential field components. Once the matrix $T$ is known (Appendix A), (11) constitutes an homogeneous equation system for $E_{o s}, E_{e s}, E_{o c}$, and $E_{e c}$, which yields the eigenvalue equation. After straightforward manipulation one arrives at

$$
A_{e} B_{o} n_{o s}^{2} \sin ^{2} \theta-A_{o} B_{e} \gamma_{o s}^{2} \cos ^{2} \theta=0
$$

where

$$
\begin{aligned}
A_{o}= & {\left[\frac{\gamma_{o s}}{\alpha_{f}}+\frac{\gamma_{c}}{\alpha_{f}}\right] \cos \left(\alpha_{f} k_{0} D\right) } \\
& -\left[1-\frac{\gamma_{o s}}{\alpha_{f}} \frac{\gamma_{c}}{\alpha_{f}}\right] \sin \left(\alpha_{f} k_{0} D\right) \\
B_{o}= & {\left[\frac{n_{o s}^{2}}{n_{f}^{2}} \frac{\alpha_{f}}{\gamma_{o s}}+\frac{n_{c}^{2}}{n_{f}^{2}} \frac{\alpha_{f}}{\gamma_{c}}\right] \cos \left(\alpha_{f} k_{0} D\right) } \\
& +\left[1-\frac{n_{o s}^{2}}{n_{f}^{2}} \frac{\alpha_{f}}{\gamma_{o s}} \frac{n_{c}^{2}}{n_{f}^{2}} \frac{\alpha_{f}}{\gamma_{c}}\right] \sin \left(\alpha_{f} k_{0} D\right) \\
A_{e}= & A_{o}\left[\gamma_{o s} \longrightarrow \gamma_{e s}\right] \\
B_{e}= & B_{o}\left[\gamma_{o s} \longrightarrow \gamma_{o s}^{2} / \gamma_{e s}\right]
\end{aligned}
$$

with

$$
\alpha_{f}=\left[n_{f}^{2}-N^{2}\right]^{1 / 2} .
$$

At $\theta=0^{\circ}$, the first term in (12) disappears, whereas $A_{o}$ and $B_{e}$ yield the eigenvalue equation for TE-polarized pure ordinary modes and TM-polarized predominantly ordinary modes, respectively. Conversely, at $\theta=90^{\circ}$, the second term vanishes and $A_{e}$, and $B_{o}$ respectively yield the eigenvalue equation for TE-polarized pure extraordinary modes and TMpolarized pure ordinary modes. At the limiting $D / \lambda=0,(12)$ 
reduces

$$
\tan ^{2} \theta=\frac{\gamma_{o s}}{n_{o s}^{2}} \frac{\left(\gamma_{c}+\gamma_{o s}\right)}{\left(\gamma_{c}+\gamma_{e s}\right)} \frac{\left(n_{o s}^{2} \gamma_{c} \gamma_{e s}+n_{c}^{2} \gamma_{o s}^{2}\right)}{\left(n_{o s}^{2} \gamma_{c}+n_{c}^{2} \gamma_{o s}\right)}
$$

which is the eigenvalue equation for the surface waves guided by the substrate-cover dielectric interface reported by D'yakonov.

\section{B. Cutoff Loci}

The effective indices of the allowed stationary guided modes are obtained as the roots of the eigenvalue equation verifying $N>n_{c}, N>n_{o s}$, and $N>n_{e s}(\theta)$. When $n_{e s}>n_{o s}>n_{c}$, guided propagation requires $N>n_{e s}(\theta)$, hence existence of totally guided modes is governed by the so-called extraordinary-cutoff for radiation to the substrate [2]. Usual waveguide modes fulfill this requirement, but there is a cutoff thickness (in units of $\lambda$ ) for the guided waves to be allowed. On the contrary, in a properly tailored waveguide having $n_{e s}>n_{c}>n_{o s}$, guided solutions occur above both the extraordinary-cutoff for radiation to the substrate and the usual cutoff for radiation to the cover, therefore guided propagation requires $N>n_{e s}(\theta)$ and $N>n_{c}$. The point is that such kind of guided waves exist for any value of the ratio $D / \lambda$, provided that a suitable orientation of the crystal optical axis is taken. This is true even for $D / \lambda=0$, then the waves being guided by the cover-substrate dielectric interface.

For the following, it is worth writing (12) and (18) as $F(N, \nu)=0$, where $\nu$ stands for all the involved waveguide parameters. Then, the allowed regions in a generic $\nu-\theta$ diagram for the waveguide modes to exist are obtained by drawing on it the critical optical axis orientations which yield the cutoff loci. These loci come from the conditions

$$
F\left(N=n_{c} ; \theta=\theta_{1}, \nu\right)=0
$$

and

$$
F\left[N=n_{e s}(\theta) ; \theta=\theta_{2}, \nu\right]=0 .
$$

In next section we will investigate the behavior of $\theta_{1}$ and $\theta_{2}$ as a function of the waveguide thickness, and the cover and the film refractive indices.

\section{DISCUSSION}

First, we focus on the existence conditions of the hybrid guided waves in ultrathin films. Fig. 2 shows the allowed and forbidden values of the optical axis orientation for the guided waves to exist, as a function of the ratio $D / \lambda$. As was pointed out in [4], when $D / \lambda=0$, the allowed angular interval is nearly centered at

$$
\theta_{0}=\sin ^{-1}\left[\frac{n_{e s}}{n_{c}} \sqrt{\frac{n_{c}^{2}-n_{o s}^{2}}{n_{e s}^{2}-n_{o s}^{2}}}\right]
$$

which is the orientation verifying $n_{e s}\left(\theta_{0}\right)=n_{c}$. This interval is extremely narrow, $\Delta \theta \equiv \theta_{2}-\theta_{1} \simeq 2^{\circ} \times 10^{-3}$, but it increases fast with the film thickness. Fig. 3 show the values reached by $\Delta \theta$ as a function of the cover refractive index, for various typical birefringent media (crystal quartz, crystal urea

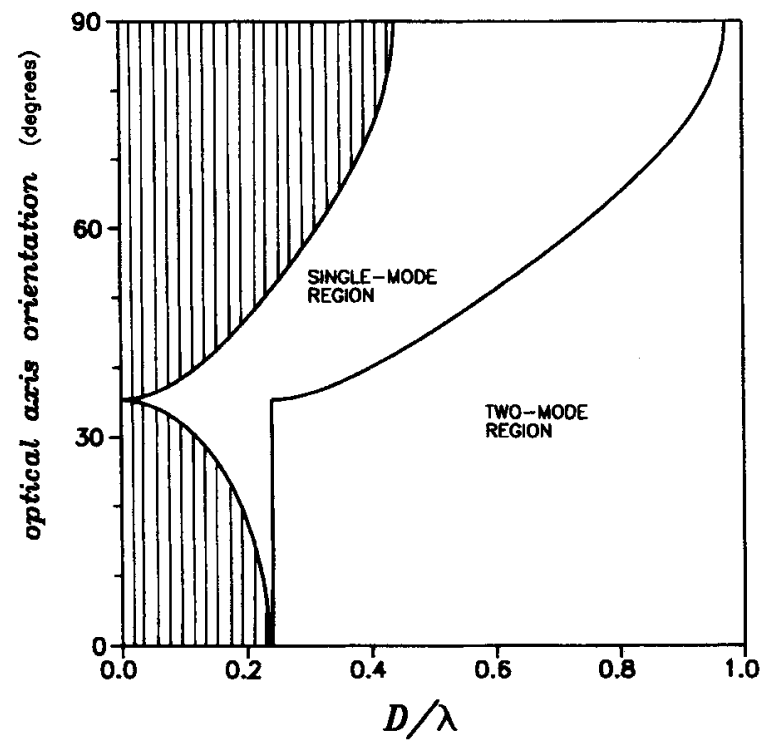

Fig. 2. Allowed optical axis orientations for the hybrid guided waves to exist as a function of the ratio $D / \lambda$. Hatched regions: forbidden values.

and $5 \mathrm{CB}$ nematic liquid crystal). As $D / \lambda$ grows, $\theta_{1} \rightarrow 0^{\circ}$ and $\theta_{2} \rightarrow 90^{\circ}$, with the limiting values being reached at the cutoff thicknesses of the $\mathrm{TE}_{0}$ wave taking place at these symmetric orientations. One has

$$
\frac{D}{\lambda}=\frac{1}{2 \pi \sqrt{n_{f}^{2}-n_{c}^{2}}} \tan ^{-1} \sqrt{\frac{n_{c}^{2}-n_{o s}^{2}}{n_{f}^{2}-n_{c}^{2}}}
$$

at $\theta=0^{\circ}$, and

$$
\frac{D}{\lambda}=\frac{1}{2 \pi \sqrt{n_{f}^{2}-n_{e s}^{2}}} \tan ^{-1} \sqrt{\frac{n_{e s}^{2}-n_{c}^{2}}{n_{f}^{2}-n_{e s}^{2}}}
$$

at $\theta=90^{\circ}$. In Fig. 2, the two-mode regime starts at a film thickness close to

$$
\frac{D}{\lambda}=\frac{1}{2 \pi \sqrt{n_{f}^{2}-n_{c}^{2}}} \tan ^{-1}\left[\frac{n_{f}^{2}}{n_{o s} n_{e s}} \sqrt{\frac{n_{c}^{2}-n_{o s}^{2}}{n_{f}^{2}-n_{c}^{2}}}\right]
$$

which is the cutoff value of the $\mathrm{TM}_{0}$ mode taking place at $\theta=$ $0^{\circ}$. Finally, taking a suitable optical axis orientation, singlemode totally guided propagation will occur for film thicknesses below

$$
\begin{aligned}
\frac{D}{\lambda}= & \frac{1}{2 \pi \sqrt{n_{f}^{2}-n_{e s}^{2}}}\left\{\tan ^{-1}\left[\frac{n_{f}^{2}}{n_{c}^{2}} \sqrt{\frac{n_{e s}^{2}-n_{c}^{2}}{n_{f}^{2}-n_{e s}^{2}}}\right]\right. \\
& \left.+\tan ^{-1}\left[\frac{n_{f}^{2}}{n_{o s}^{2}} \sqrt{\frac{n_{e s}^{2}-n_{o s}^{2}}{n_{f}^{2}-n_{e s}^{2}}}\right]\right\} .
\end{aligned}
$$

Above these critical thicknesses, usual waveguide modes take place [1], [2], yielding the $\left[\mathrm{TE}_{0}, \mathrm{TE}_{0}\right]$ and the $\left[\mathrm{TM}_{0}\right.$, $\mathrm{TM}_{0}$ ] hybrid modes.

Fig. 4 shows the allowed region in the $n_{c}-\theta$ diagram for the guided waves to exist, for a film thickness $D / \lambda=0.2$. The single-mode region takes place for cover refractive index larger that the critical value given by (24). Finally, the allowed 


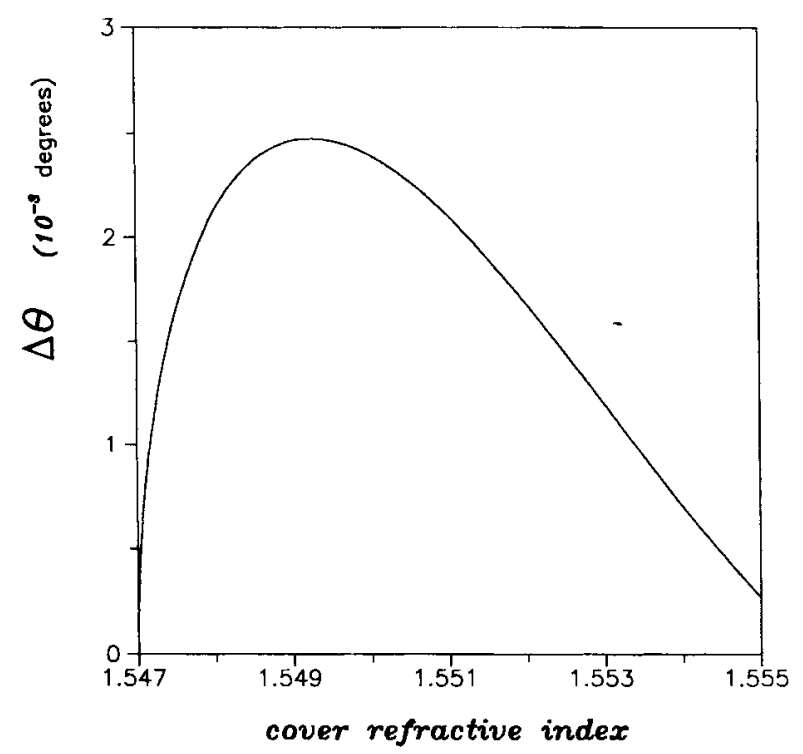

(a)

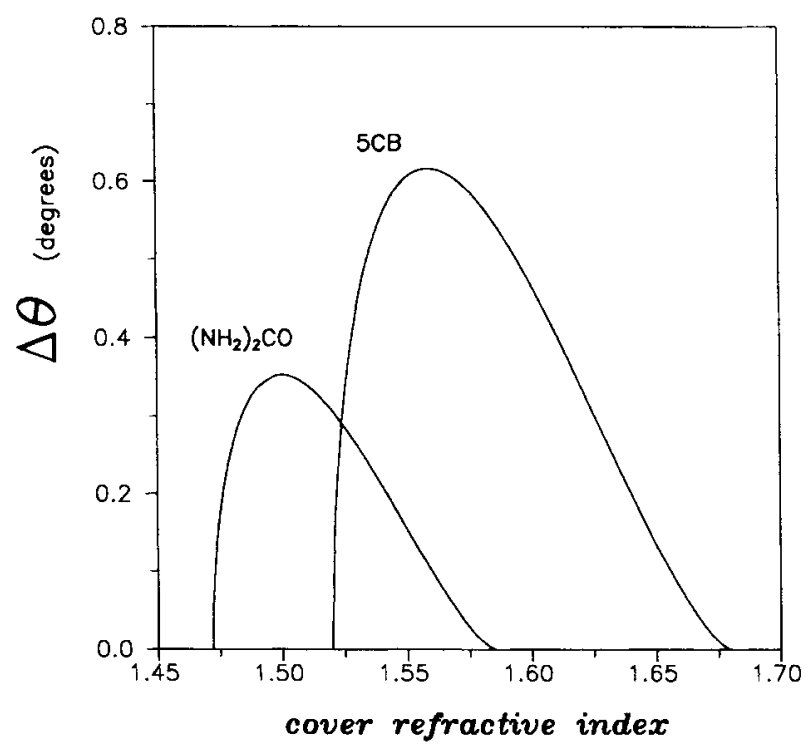

(b)

Fig. 3. Width of the allowed angular range for the surface wave guided by the glass-crystal interface $(D / \lambda=0)$ to exist, as a function of the glass refractive index. (a) Crystal quartz; (b) $5 \mathrm{CB}$ nematic liquid crystal, (4-cyano-4' -pentylbiphenyl, $n_{o} \simeq 1.52, n_{\epsilon} \simeq 1.68$ ) and crystal urea, $\left[\left(\mathrm{NH}_{2}\right)_{2} \mathrm{CO}, n_{o} \simeq 1.4720, n_{e} \simeq 1.5852\right)$.

optical axis orientations as a function of the film refractive index are shown in Fig. 5, for a thinner film of thickness $D / \lambda=0.1$.

In Fig. 6 we have plotted the effective index of the allowed guided waves as a function of the optical axis orientation, for two different film thicknesses, both belonging to the singlemode region. As expected from coupled-mode approach since $\epsilon_{y y} \gg \epsilon_{x y}$, the plot shows that the allowed hybrid guided mode behaves as a weakly-perturbed pure $\mathrm{TE}_{0}$-polarized wave propagating in an "isotropic" structure having $n_{s}=n_{e s}(\theta)$. The eigenvalue equation of such a mode is just given by $A_{e}=$ 0 . This equation yields approximate numerical values of $N$ and $\theta_{2}$, and an approximate closed-from expression of the cutoff

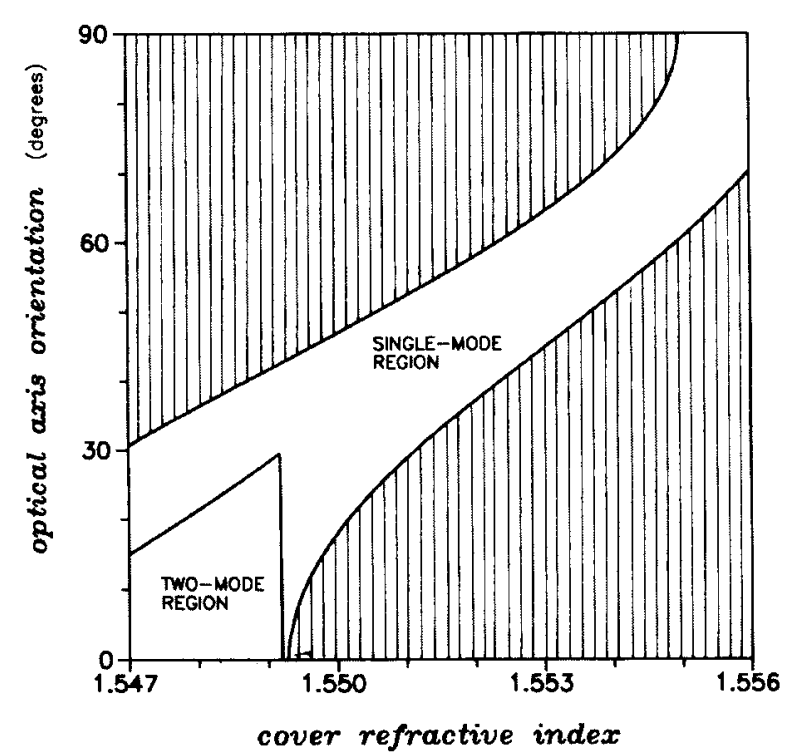

Fig. 4. Allowed optical axis orientations as a function of the cover refractive index. Here $D / \lambda=0.2$.

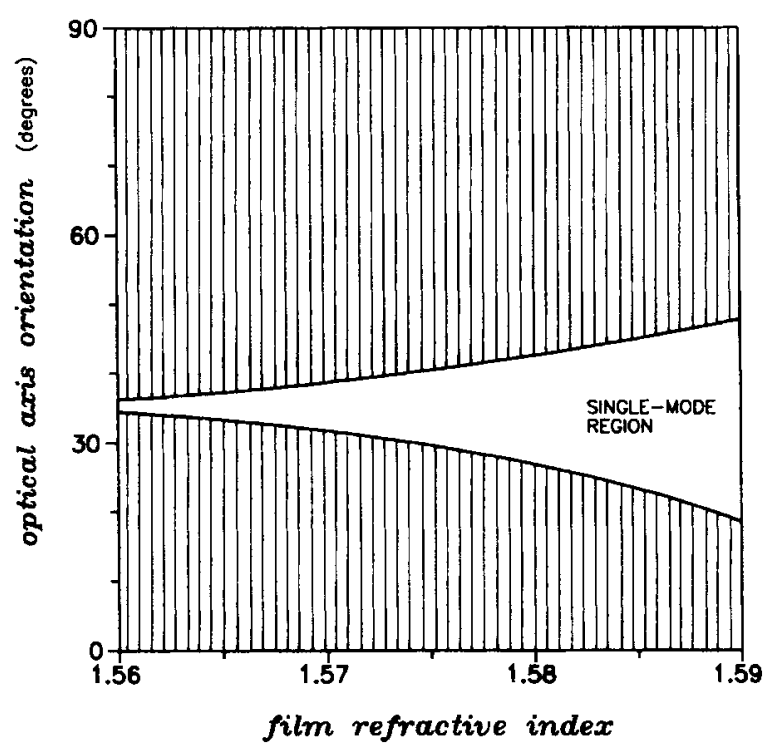

Fig. 5. Allowed optical axis orientations as a function of the film refractive index. Here $D / \lambda=0.1$.

optical axis orientation for radiation to the cover. One arrives at

$$
\begin{aligned}
\sin ^{2} \theta_{1} \simeq & \frac{n_{e s}^{2}}{n_{e s}^{2}-n_{o s}^{2}} \\
& \cdot\left[1-\frac{n_{o s}^{2}}{n_{c}^{2}-\left(n_{f}^{2}-n_{c}^{2}\right) \tan ^{2}\left(k_{0} D \sqrt{n_{f}^{2}-n_{c}^{2}}\right)}\right] .
\end{aligned}
$$

Likewise, in the two-mode region, the second allowed hybrid wave has an almost TM nature. Accordingly, as a function of the optical axis orientation, it approximately behaves as a pure ordinary $\mathrm{TM}_{0}$ mode.

Fig. 7 shows the $E_{y}$ and $E_{x}$ transverse field profiles of the hybrid wave guided by an structure with $D=0.1 \lambda$. When considering the surface waves guided by the cover- 


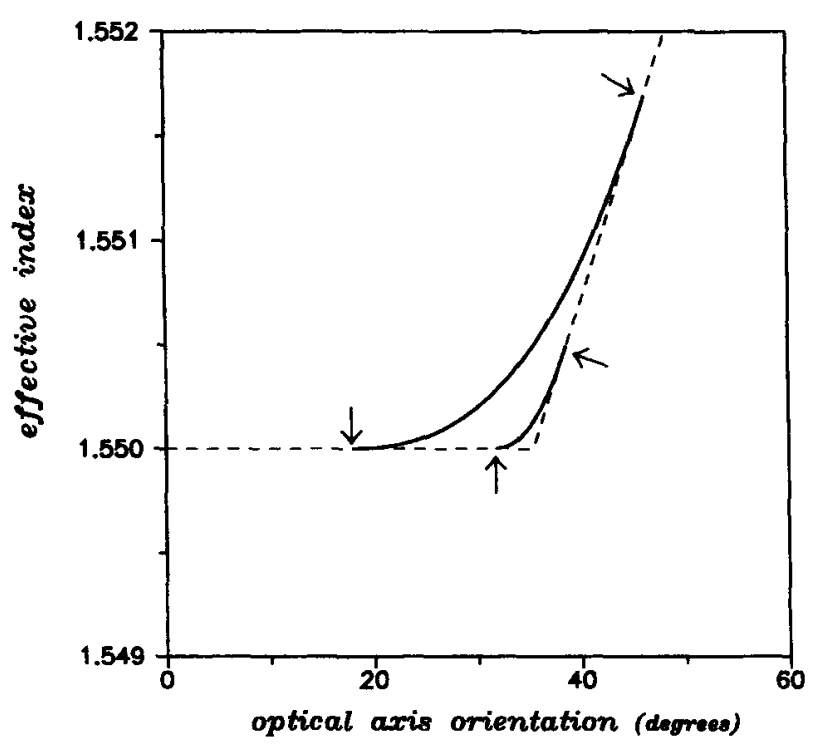

Fig. 6. Effective index of the TE-dominant hybrid mode as a function of the optical axis orientation, for $D / \lambda=0.1$ and $D / \lambda=0.2$. Dashes show the cutoff lines $N=n_{c}$ and $N=n_{e s}(\theta)$, and the arrows indicate the cutoff points.

substrate interface, the maximum value of $E_{y}$ takes place at the birefringent medium. The location of this maximum moves away from the interface as the optical axis orientation approaches the cutoff value $\theta_{2}$, but enters the film very fast as the film thickness grows.

The nature of the hybrid mode in terms of transverse and longitudinal components is displayed in Fig. 8, showing the ratio

$$
R \equiv\left|\frac{E_{y}(z)}{E_{x}(z)}\right|_{z=0}
$$

between the field components of the fundamental mode, as a function of the optical axis orientation, for different values of the film thickness. When considering the surface waves guided by the cover-substrate interface $(D / \lambda=0)$, this expression yields

$$
R \simeq \frac{n_{c}^{2}}{\gamma_{c} \gamma_{o s}} \tan \theta \sim 10^{5}
$$

Likewise, Figs. 7 and 8 show that in the thin film case $\left|E_{y}\right| \gg\left|E_{x}\right|$, also. This drives us to the conclusion that the hybrid guided waves we are dealing with are almost transverse.

A deeper insight into the nature of the allowed hybrid guided waves can be obtained by computing the carried power flow. This guided wave power, per unit length along the $y$ dimension, comes from the integration of the Poynting vector over the cross section of the waveguide

$$
P=\int_{-\infty}^{\infty} \vec{S}(z) \cdot \hat{x} \mathrm{~d} z=P_{\mathrm{TE}}+P_{\mathrm{TM}}
$$

with

$$
\begin{aligned}
S_{x}(z) & =\frac{1}{2 \eta}\left[E_{y}(z) H_{z}(z)-E_{z}(z) H_{y}(z)\right] \\
& =S_{x, \mathrm{TE}}+S_{x, \mathrm{TM}}
\end{aligned}
$$
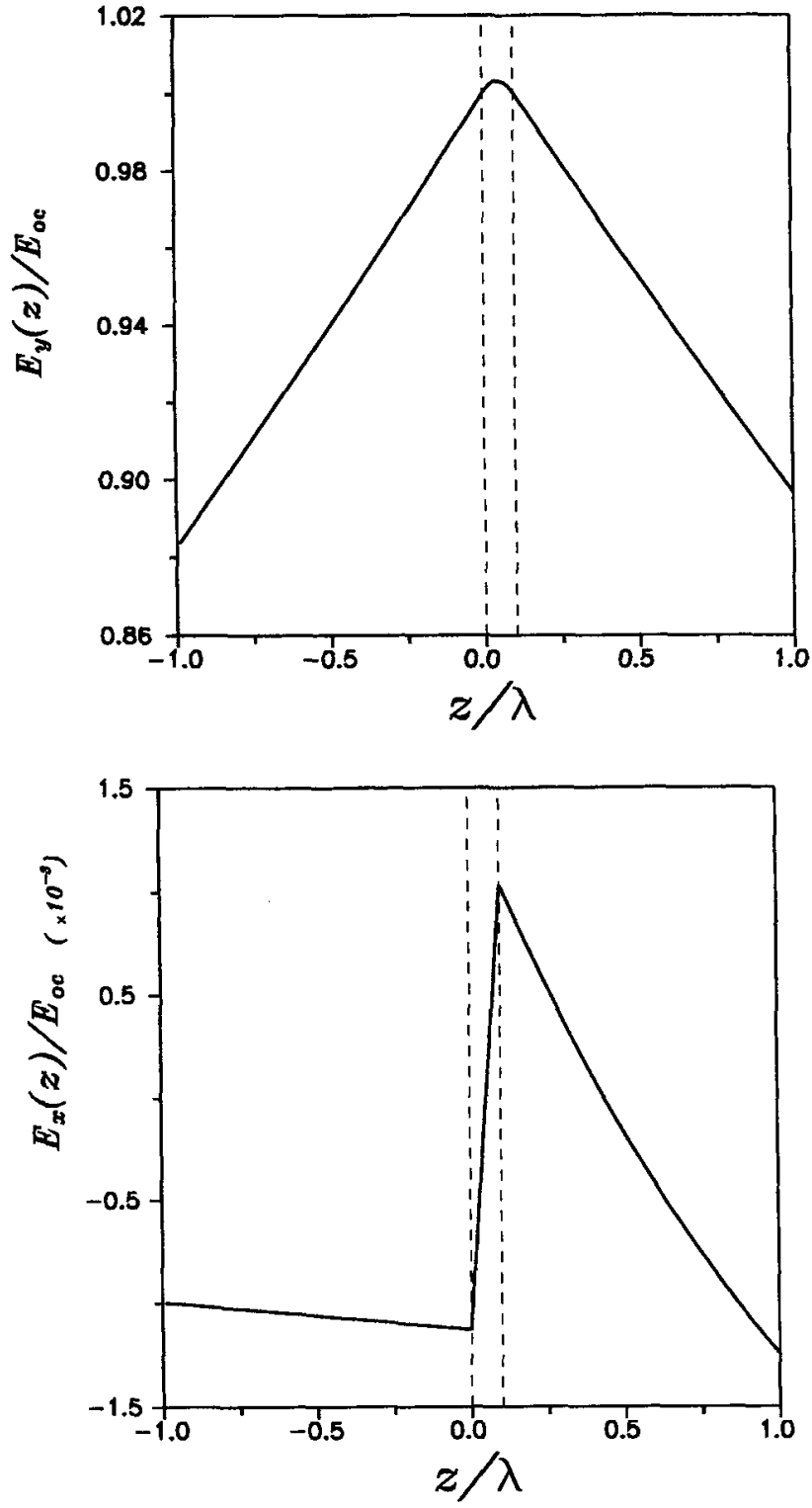

Fig. 7. Transverse field profiles $D / \lambda=0.1$.

Attention will be focussed on the TE and TM fraction of total power flow. In the case $D / \lambda=0$, one gets

$$
\Gamma_{\mathrm{TE}} \equiv \frac{P_{\mathrm{TE}}}{P} \simeq \frac{\left(\gamma_{c}+\gamma_{e s}\right) n_{c}^{2} \tan ^{2} \theta}{\gamma_{e s} \gamma_{o s}^{2}+\left(\gamma_{c}+\gamma_{e s}\right) n_{c}^{2} \tan ^{2} \theta} .
$$

Substitution of the numerical values of various involved parameters yields $\Gamma_{\mathrm{TE}} \simeq 1$, and $\Gamma_{\mathrm{TM}}\left(\theta_{0}\right) \simeq 4 \times 10^{-3}$. When considering the wave guided by an ultrathin film, similar values are obtained (typically, $E_{y} \sim 10 E_{z}$, hence $P_{\mathrm{TE}} \sim$ $10^{2} P_{\mathrm{TM}}$, and $\Gamma_{\mathrm{TE}} \sim 0.99$ ), pointing out the TE-dominant nature of the hybrid mode.

Finally, since we are dealing with waves guided by either an interface or a very thin film, the degree of confinement of the waves becomes a salient point that must be addressed. To this aim, in Fig. 9 we have plotted the effective waveguide thickness $(W)$ of the allowed hybrid guided wave as a function of the optical axis orientation, for different values of the film thickness. Due to the birefringent nature of the involved material, the field decay rate at the substrate amount to 


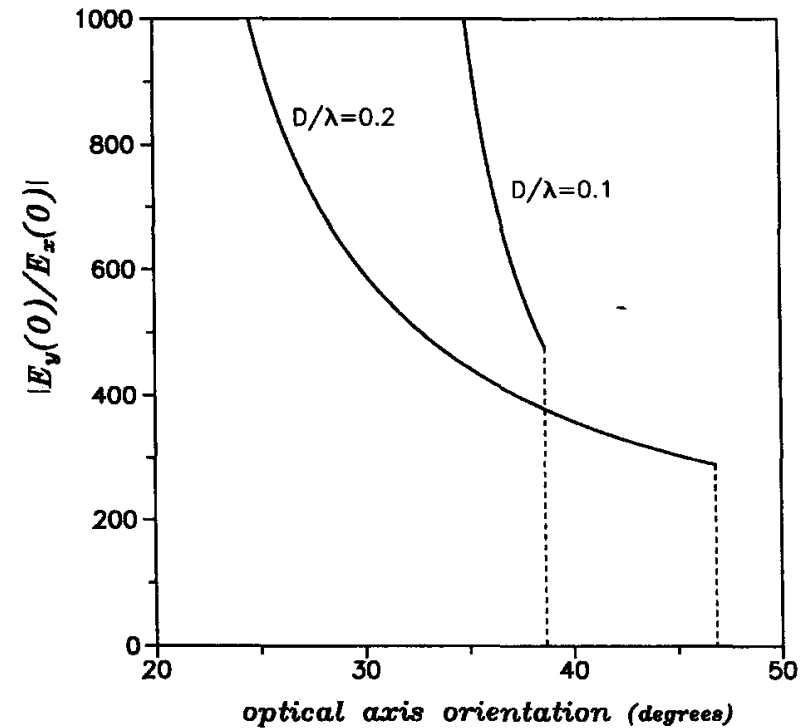

Fig. 8. Ratio between transverse $(\hat{y})$ and longitudinal electric field components at the cover-film interface, as a function of the optical axis orientation. Dashes indicate the cutoff line $N=n_{e s}(\theta)$.

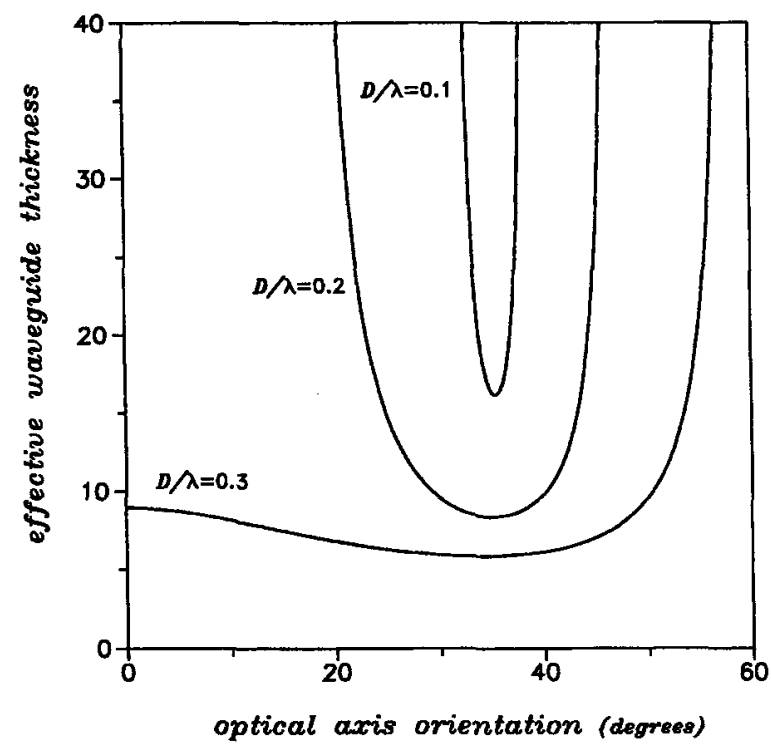

Fig. 9. Effective waveguide thickness (in units of $\lambda$ ) as a function of the optical axis orientation.

different values for each field component. Nevertheless, since the hybrid guided wave is TE-dominant, the quantity

$$
W \equiv \frac{D}{\lambda}+\frac{1}{2 \pi}\left[\frac{1}{\gamma_{c}}+\frac{1}{\gamma_{e s}}\right]
$$

is a good measure of the actual effective waveguide thickness. $W$ diverges at the cutoff loci but, according to the plot, amount to small values near $\theta_{0}$.

\section{CONCLUSION}

To sum up, we have shown that properly tailored waveguides consisting on ultrathin films cladded by a positive birefringent crystal allow existence of a TE-dominant hybrid guided wave for waveguide parameters below usual cutoff. Our calculations refer to organic films of precisely controlled thickness in the range $50-150 \mathrm{~nm}$, which correspond to the typical values of Langmuir-Blodgett ultrathin films.

The existence conditions of the allowed guided wave have been shown to strongly depend on the waveguide parameters. This suggests that the related guided-to-radiated wave transition can be useful for switching applications requiring strong sensitive dependence on the refractive index values. Thermooptically-induced variations, by pulse heating, and purely dispersive intensity-dependent refractive index changes in properly tailored waveguides made on optically nonlinear materials are promising possibilities of current investigation $[8]$.

\section{APPENDIX: THE MATRIX $T$}

The characteristic matrix of an homogeneous dielectric film can be analytically calculated by using the $4 \times 4$ formalism [7]. In the case of an isotropic film, taking the usual ordering criterion $\Psi^{t}=\left(E_{x}, H_{y}, E_{y}, H_{x}\right)$, it simply writes

$$
T=\left(\begin{array}{cc}
U_{\mathrm{TM}} & 0 \\
0 & U_{\mathrm{TE}}
\end{array}\right)
$$

where

$$
U=\left(\begin{array}{cc}
\cos \left(\alpha_{f} k_{0} D\right) & -\delta \sin \left(\alpha_{f} k_{0} D\right) \\
\delta^{-1} \sin \left(\alpha_{f} k_{0} D\right) & \cos \left(\alpha_{f} k_{0} D\right)
\end{array}\right)
$$

Here $\delta_{\mathrm{TE}}=-1 / \alpha_{f}$, and $\delta_{\mathrm{TM}}=\alpha_{f} / n_{f}^{2}$. Accordingly, the electric field at the film region writes

$$
\begin{aligned}
& E_{f, x}(z)=E_{e c}\left[\cos \left(\alpha_{f} k_{0} z\right)-\frac{n_{c}^{2}}{n_{f}^{2}} \frac{\alpha_{f}}{\gamma_{c}} \sin \left(\alpha_{f} k_{0} z\right)\right] \\
& E_{f, y}(z)=E_{o c}\left[\cos \left(\alpha_{f} k_{0} z\right)+\frac{\gamma_{c}}{\alpha_{f}} \sin \left(\alpha_{f} k_{0} z\right)\right] \\
& E_{f, z}(z)=-E_{e c}\left[\frac{n_{c}^{2}}{n_{f}^{2}} \frac{N}{\gamma_{c}} \cos \left(\alpha_{f} k_{0} z\right)+\frac{N}{\alpha_{f}} \sin \left(\alpha_{f} k_{0} z\right)\right]
\end{aligned}
$$

\section{REFERENCES}

[1] A. Knoesen, T. K. Gaylord, and M. G. Moharam, "Hybrid guided modes in uniaxial dielectric planar waveguides," J. Lightwave Technol., vol. 6, pp. 1083-1104, 1988.

[2] L. Torner, J. Recolons, and J. P. Torres, "Guided-to-leaky mode transition in uniaxial optical slab waveguides," J. Lightwave Technol., vol. 11, pp. 1592-1600, 1993.

[3] L. Torner, J. P. Torres, and D. Mihalache, "New type of guided waves in birefringent media," IEEE Photon. Technol. Lett., vol. 5, pp. 201-203, 1993.

[4] M. I. D'yakonov, "New type of electromagnetic wave propagating at an interface," Sov. Phys. JETP, vol. 67, pp. 714-716, 1988.

[5] G. I. Stegeman, C. T. Seaton, and R. Zanoni, "Organic films in nonlinear integrated optics structures," Thin Solid Films, vol. 152, pp. 231-263, 1987.

[6] E. Van Tomme, P. P. Van Daele, R. G. Baets, and P. E. Lagasse, "Integrated optic devices based on nonlinear optical polymers," IEEE J. Quantum Electron., vol. 27, pp. 778-787, 1991.

[7] D. W. Berreman, "Optics in stratified and anisotropic media: $4 \times 4$ matrix formalism," J. Opt. Soc. Am., vol. 62, pp. 502-510, 1972.

[8] L. Torner, J. P. Torres, F. Lederer, D. Mihalache, D. M. Baboiu, and M. Ciumac, "Nonlinear hybrid waves guided by birefringent interfaces," Electron. Lett., vol. 29, pp. 1186-1188, 1993. 
Lluís Torner (M'92), photograph and biography not available at the time of publication.

Juan P. Torres, photograph and biography not available at the time of publication.
Concepción Ojeda, photograph and biography not available at the time of publication.

Dumitru Mihalache, photograph and biography not available at the time of publication. 